\title{
Transition from strong to weak coupling and the onset of lasing in semiconductor microcavities
}

\author{
R. Butté, ${ }^{1}$ G. Delalleau, ${ }^{1}$ A. I. Tartakovskii, ${ }^{1}$ M. S. Skolnick, ${ }^{1}$ V. N. Astratov, ${ }^{1}$ J. J. Baumberg, ${ }^{2}$ G. Malpuech, ${ }^{3,4}$ \\ A. Di Carlo, ${ }^{3}$ A. V. Kavokin, ${ }^{4}$ and J. S. Roberts ${ }^{5}$ \\ ${ }^{1}$ Department of Physics and Astronomy, University of Sheffield, Sheffield S3 7RH, United Kingdom \\ ${ }^{2}$ Department of Physics and Astronomy, University of Southampton, Southampton SO17 1BJ, United Kingdom \\ ${ }^{3}$ INFM-Department of Electrical Engineering, University of Rome Tor Vergata, I-00133 Roma, Italy \\ ${ }^{4}$ LASMEA (UMR 6602 CNRS), Université Blaise Pascal Clermont-II, 63177 Aubiére Cedex, France \\ ${ }^{5}$ Department of Electronic and Electrical Engineering, University of Sheffield, Sheffield S1 3JD, United Kingdom
}

(Received 30 October 2001; published 29 April 2002)

\begin{abstract}
Angular-dependent emission spectra are investigated in a strongly coupled InGaAs-GaAs-AlAs-based semiconductor microcavity as a function of excitation intensity and of detuning between the uncoupled exciton and photon modes. Under conditions of nonresonant excitation, it is shown that the onset of stimulated emission always occurs in the weak coupling regime. Angular-dependent studies show that the transition to weak coupling occurs when the linewidth of high $k$ excitons becomes of the order of the normal mode splitting of the exciton-polariton coupled modes. We conclude that, under nonresonant excitation, "polariton lasing," where stimulated polariton scattering followed by photon emission occurs in the strong coupling regime, is only likely to be achieved in systems with larger exciton binding energy than in GaAs-based structures or possibly also in GaAs microcavities containing excess concentrations of free electrons. The experimental results below threshold are found to be in good agreement with numerical solution of Boltzmann kinetic equations for the photoexcited polariton distribution.
\end{abstract}

DOI: 10.1103/PhysRevB.65.205310

PACS number(s): 78.67. $-\mathrm{n}, 71.36 .+\mathrm{c}, 42.55 . \mathrm{Sa}, 78.45 .+\mathrm{h}$

\section{INTRODUCTION}

Semiconductor microcavities permit the control of the interactions between quantum well excitons and cavityconfined photons. Most importantly the resulting excitonphoton coupled modes (exciton-polaritons) exhibit novel features possessed by neither excitons nor photons alone. ${ }^{1}$ Of particular interest for the present work, the density of states in the strongly coupled polariton region is very small, of order $10^{-4}$ of that of the excitons. As a result it is expected to be very much easier than in bulk materials to achieve state occupancies exceeding unity, and thus to achieve final state stimulation of scattering from the high density of photocreated excitons in the exciton reservoir into the $k \approx 0$ lightemitting polariton states [see schematic diagram in Fig. 1(a)]. The $k \approx 0$ states have significant photon character and are rapidly transformed into external photons, on time scales determined by the photon lifetime in the cavity, of order 1-10 ps in high finesse cavities. We have recently reported the observation of such stimulated polariton scattering, ${ }^{2-4}$ arising from the bosonic character of the polariton quasiparticles, in resonant excitation conditions where polaritons are injected directly into the lower polariton dispersion curve, at the point of inflection of the lower polariton branch [see Fig. 1(b)]. Such resonant excitation permits direct population of the low density of states, strongly coupled polariton region, and allows state occupancies greater than one to be achieved, at total injected densities two to three orders of magnitude below the densities at which exciton screening, and the loss of strong coupling occurs.

The purpose of the present paper is to investigate whether stimulated scattering can be achieved under conditions of nonresonant excitation in GaAs-based microcavities, similar to the conditions under which a real semiconductor device under electrical injection may be expected to operate. ${ }^{5}$ Under nonresonant conditions, excitons are injected at energies above the stop band of the Bragg mirrors of the microcavity. The excitons relax rapidly by LO phonon emission to populate the exciton reservoir, from where, at low density, they emit acoustic phonons in times of order 1 nsec, before escaping from the cavity on time scales of $\sim 1$ ps by photon leakage through the Bragg mirrors [see schematic in Fig. 1(a)]. The relatively slow relaxation compared to the very fast escape leads to the formation of a relaxation bottleneck for the polaritons for negative detunings, ${ }^{6-9}$ with strongly depleted low $k$ states compared to the states in the resonance regime, and in the exciton reservoir [Fig. 1(a)]. The occurrence of the bottleneck inhibits very strongly the achievement of high state occupancies in the near $k=0$ region. However, we have also shown that with increasing power the bottleneck can be suppressed by exciton-exciton scattering from the high $k$ states towards $k=0$, whilst at the same time remaining in the strong coupling regime. ${ }^{6}$

In the present paper, we investigate the modification of the bottleneck, and the onset of stimulated emission in a series of nonresonantly excited photoluminescence experiments on a GaAs-based microcavity. A wide range of negative detunings, the energy separation between the uncoupled excitons and photons, is investigated to study the influence of the exciton/photon character of the low $k$ states on (a) the transition from strong to weak coupling, (b) the strength of the bottleneck and whether it can be suppressed, and (c) the onset and character of strongly nonlinear, stimulated emission. Most importantly angular dependent studies are employed to provide a stringent test of whether there is departure from strong coupling at high excitation intensity. It is found that in typical GaAs-based microcavities, the onset of gain and strong line narrowing always corresponds to con- 

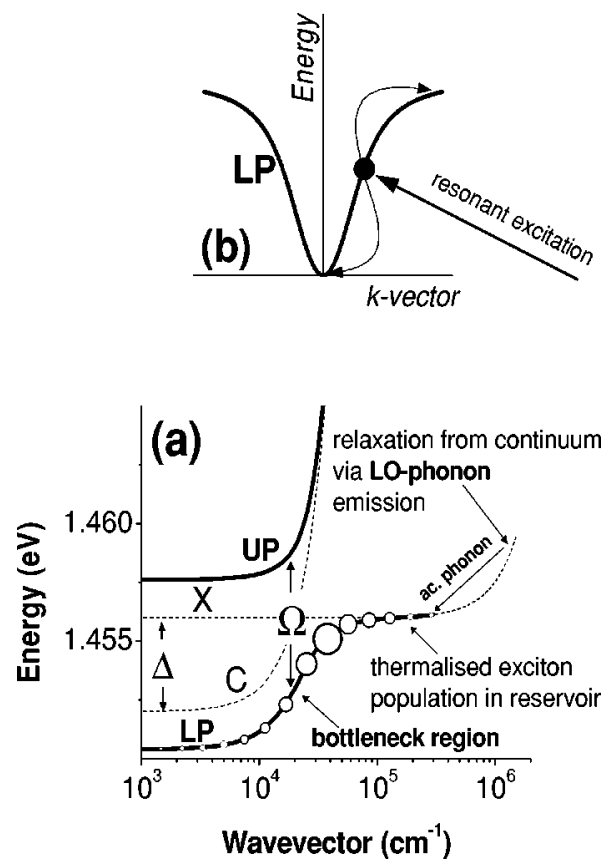

FIG. 1. (a) Schematic diagram of microcavity dispersion in the strong coupling regime for small negative detuning between the uncoupled exciton and cavity modes. Both uncoupled cavity photon and exciton (dashed), and the strongly coupled polariton modes (thick) are shown. In the strong coupling regime, the states have mixed exciton-photon character at low $k$, whereas with increasing $k$, the lower polariton states become increasingly excitonlike. Following nonresonant excitation, excitons relax by LO phonon emission into the reservoir. Competition between slow acoustic phonon relaxation and fast escape from the cavity leads to the formation of the relaxation bottleneck, with the size of the circles representing schematically the polariton population as a function of $k$. (b) Lower polariton branch dispersion on linear scale, for reference, showing resonant excitation at the point of inflection of the lower polariton branch.

ventional vertical cavity lasing in the weak coupling regime. From studies of the linewidths of the high $k$ states it is shown that the strong coupling is lost at exciton densities which give rise to broadening of the exciton lines of the order of the normal mode splitting of the polariton coupled modes.

An important feature of the present work is its focus on studies for negative detuning, where the uncoupled cavity mode lies to lower energy than the uncoupled excitons. For zero and positive detunings a relaxation bottleneck is not observed, ${ }^{6,7}$ probably due to effective disorder scattering from the uncoupled exciton levels, or possibly also because of efficient polariton scattering by residual free carriers. ${ }^{10} \mathrm{In}$ these cases, the $k=0$ emission behaves in a very similar way to that of the uncoupled excitons, with sublinear increases of the $k=0$ intensities with power, up to the threshold for stimulated emission. ${ }^{11}$ By employing negative detuning, it is likely that the photon character of the $k=0$ states will be more prominent, with greater probability of the desired inelastic scattering from the exciton reservoir, as opposed to direct elastic scattering from the tail of the exciton distribution. We also present a theoretical model based on the Boltzmann kinetic equations for exciton polaritons in two- dimensional reciprocal space to achieve a more profound understanding of the physical mechanisms underlying the observed polariton distributions. Good semiquantitative agreement between the theoretical results and the experimental data below threshold is obtained. Strong support for the key role of exciton-exciton scattering in determining the polariton distributions at densities below the screening threshold is obtained.

As a result of the results presented we are able to provide definitive answers to the question of whether "polariton lasing" (sometimes termed "boser" emission) ${ }^{12}$ can be achieved in GaAs-based microcavities. This area has been the subject of much controversy in the past with observation of boser emission being claimed, ${ }^{13}$ and then retracted, ${ }^{14}$ and furthermore being shown both theoretically and experimentally to be due to lasing in the weak coupling regime (for a sample with positive detuning). ${ }^{15}$ The results presented here are a major extension of our work in Ref. 6 where only one small negative detuning was studied, and power densities below the onset of strong nonlinear emission and line narrowing were employed.

The paper is organized in the following way. Following this introduction, the sample and experimental setup are described. This is followed in Sec. III A by presentation of the spectra at $k=0$ from well below to above the stimulation threshold, for three negative detunings exhibiting markedly different properties. In Sec. III B, the angular dependence of the spectra is presented over the full range of powers. Section III C presents the theoretical approach we use to describe the polariton dynamics in the strong-coupling regime, and then in Sec. III D the dispersion curves and polariton linewidths as a function of $k$ are described. From these results, the variation of the polariton distribution with power and how it depends on detuning, and the transition from weak to strong coupling and its relation to the stimulation threshold, are deduced. The impact of exciton-exciton scattering on the polariton kinetics is revealed by comparison of the experimental data with the results of the theoretical model. Finally in Sec. IV, the main conclusions are summarized.

\section{EXPERIMENTAL DETAILS}

The experiments were performed on a $3 \lambda / 2$ InGaAs/ GaAs/AlAs microcavity containing two pairs of three $\mathrm{In}_{0.06} \mathrm{Ga}_{0.94} \mathrm{As}$ quantum wells embedded at the antinodes of the optical field of the GaAs cavity. The cavity was surrounded by 17 (upper) and 20 (lower) repeats of $\mathrm{Al}_{0.13} \mathrm{Ga}_{0.87} \mathrm{As} / \mathrm{AlAs}$ Bragg mirrors. The experiments were carried out in reflection geometry in a cold finger cryostat permitting wide angular access up to $\pm 60^{\circ}$. Photoluminescence (PL) was excited by $1.59 \mathrm{eV} \mathrm{cw}$ radiation from a Ti:sapphire laser focussed to a $25 \mu \mathrm{m}$ spot, collected with angular resolution of $1^{\circ}$ by a fiber mounted on a rotating rail and detected by a monochromator, nitrogen cooled CCD combination. The spot size was measured directly by moving a pinhole across the focal plane of the beam, and monitoring the power transmitted. All the results presented here were obtained at a sample temperature of $25 \mathrm{~K}$ (measured by a 


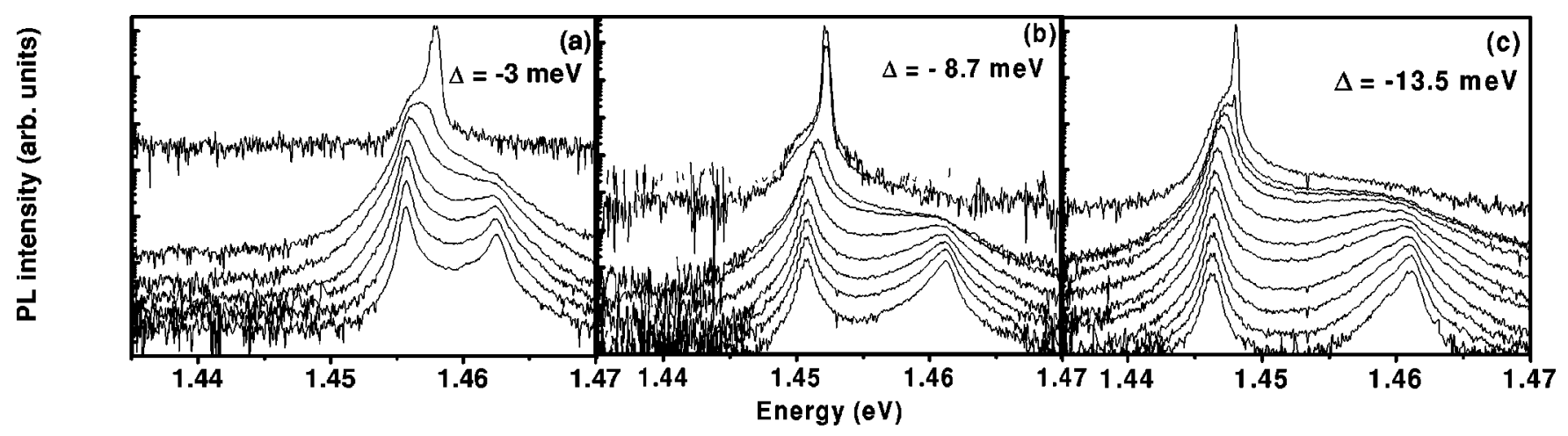

FIG. 2. (a)-(c) PL spectra measured at $k=0$ as a function of power for detunings $\Delta$ of $-3,-8.7$, and $-13.5 \mathrm{meV}$. In each case the lowest spectrum is taken at $4 \mathrm{~W} / \mathrm{cm}^{2}$. The onset of strong line narrowing occurs at 400,800 , and $1600 \mathrm{~W} / \mathrm{cm}^{2}$, respectively, in (a) $-(\mathrm{c})$, respectively.

temperature sensor glued to the sample surface), although very similar results were obtained in the range 10 to $50 \mathrm{~K}$.

\section{EXPERIMENTAL RESULTS AND DISCUSSION}

\section{A. Spectra detected at $\theta=0, k=0$. The transition from} spontaneous photoluminescence to stimulation

A series of spectra as a function of incident power for three negative detunings $(\Delta)$ between cavity and exciton modes of $-3,-8.7$, and $-13.5 \mathrm{meV}$ is presented in Figs. 2(a), 2(b), 2(c), respectively (the lowest powers in each case are $4 \mathrm{~W} / \mathrm{cm}^{2}$, and range up to 400,1200 , and $3200 \mathrm{~W} / \mathrm{cm}^{2}$ in Figs. 2(a), 2(b), 2(c), respectively). The powers quoted are estimated powers absorbed in the cavity, allowing for $50 \%$ attenuation in the Bragg mirrors and $50 \%$ absorption by the cavity. In each case, at low powers two PL peaks from the upper (UPB) and lower (LPB) polariton branches are observed, with the separation in energy between the peaks increasing with increasing detuning. With increasing power, very little change in the form of the spectra is observed up to $\sim 40 \mathrm{~W} / \mathrm{cm}^{2}$. Above such powers the UPB signal is observed to broaden, followed by a small upshift of the LPB signal. Finally above threshold power densities of 400, 800, and $1600 \mathrm{~W} / \mathrm{cm}^{2}$ in Figs. 2(a), 2(b), 2(c) respectively, very strong line narrowing from the linewidths of $0.5 \mathrm{meV}$ at low power to $0.2 \mathrm{meV}$ limited by the spectrometer resolution, ${ }^{16}$ accompanied by a strong nonlinear increase of intensity is observed. ${ }^{17} \mathrm{Up}$ to the onset of the strong line narrowing, the PL intensities increase superlinearly with power, as shown in Fig. 3, as reported previously by Senellart et al. ${ }^{18}$ and by Tartakovskii et al., ${ }^{6}$ consistent with increased population of the low $k$ states by exciton-exciton pair scattering. 6,9 However, the main interest of the present paper is the nature of the phenomena underlying the strong line narrowing and the much faster nonlinear increase of intensity observed at the highest powers in Figs. 2(a), 2(b), 2(c) and in Fig. 3.

\section{B. Angular dependent studies: The bottleneck and variation with power}

In order to investigate the nonlinear behavior, and the transition from the phenomena occurring at low powers, angular dependent measurements to probe the variation of the polariton populations as a function of in-plane wave vector with increasing power were carried out. Angle resolved spectra at low power $\left(4 \mathrm{~W} / \mathrm{cm}^{2}\right)$ well below threshold are presented in Figs. 4(a), 4(b), 4(c) respectively, and at powers just above threshold in Figs. 4(d), 4(e), 4(f). LPB populations as a function of angle and hence in plane wave vector $(k)$ (since $k=\omega / c \sin \theta$ ) deduced from the results of Fig. 4 are shown in Fig. 5. The relative polariton populations are obtained from the integrated PL intensities, following correction for the photon fraction of the states as a function of $\theta$, as described previously in, e.g., Refs. 2 and 6. The main effect of the correction for the photon fraction, relative to the raw data in Fig. 4 is to increase the relative weight of the high $k$ points, since in all cases the photon fraction is a rapidly decreasing function of $k$, as the states become increasingly excitonlike at high $k$.

We first consider the low power results of Figs. 4(a)-4(c). In each case the PL intensities increase with increasing $\theta$, reach a maximum at angles close to resonance between the uncoupled exciton and cavity modes $\left(16^{\circ}, 25^{\circ}\right.$ and $28^{\circ}$ for $\Delta=-3,-8.7$, and $-13.5 \mathrm{meV}$, respectively), and then decrease again to higher angle. The depletion of the low $k$

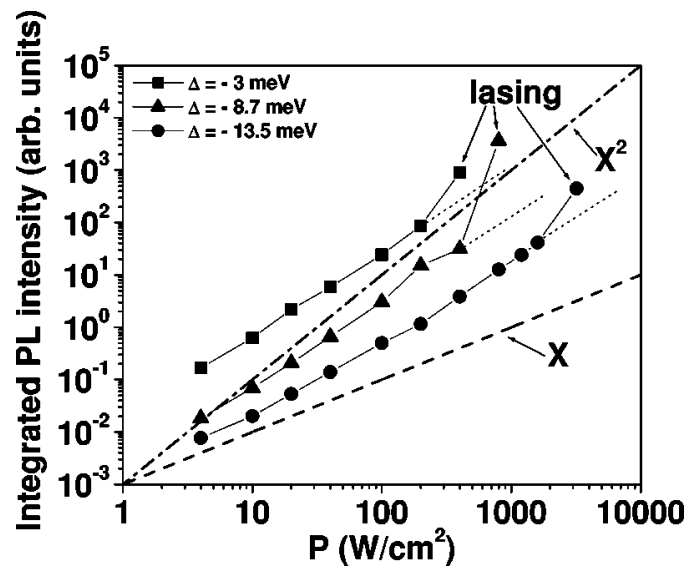

FIG. 3. Integrated PL intensity versus power density for $\Delta=-3$, -8.7 , and $-13.5 \mathrm{meV}$. In each case an approximately quadratic variation is found, consistent with pair scattering from the reservoir to lower $k$ states, up to the onset of strong line narrowing and stimulated emission. 

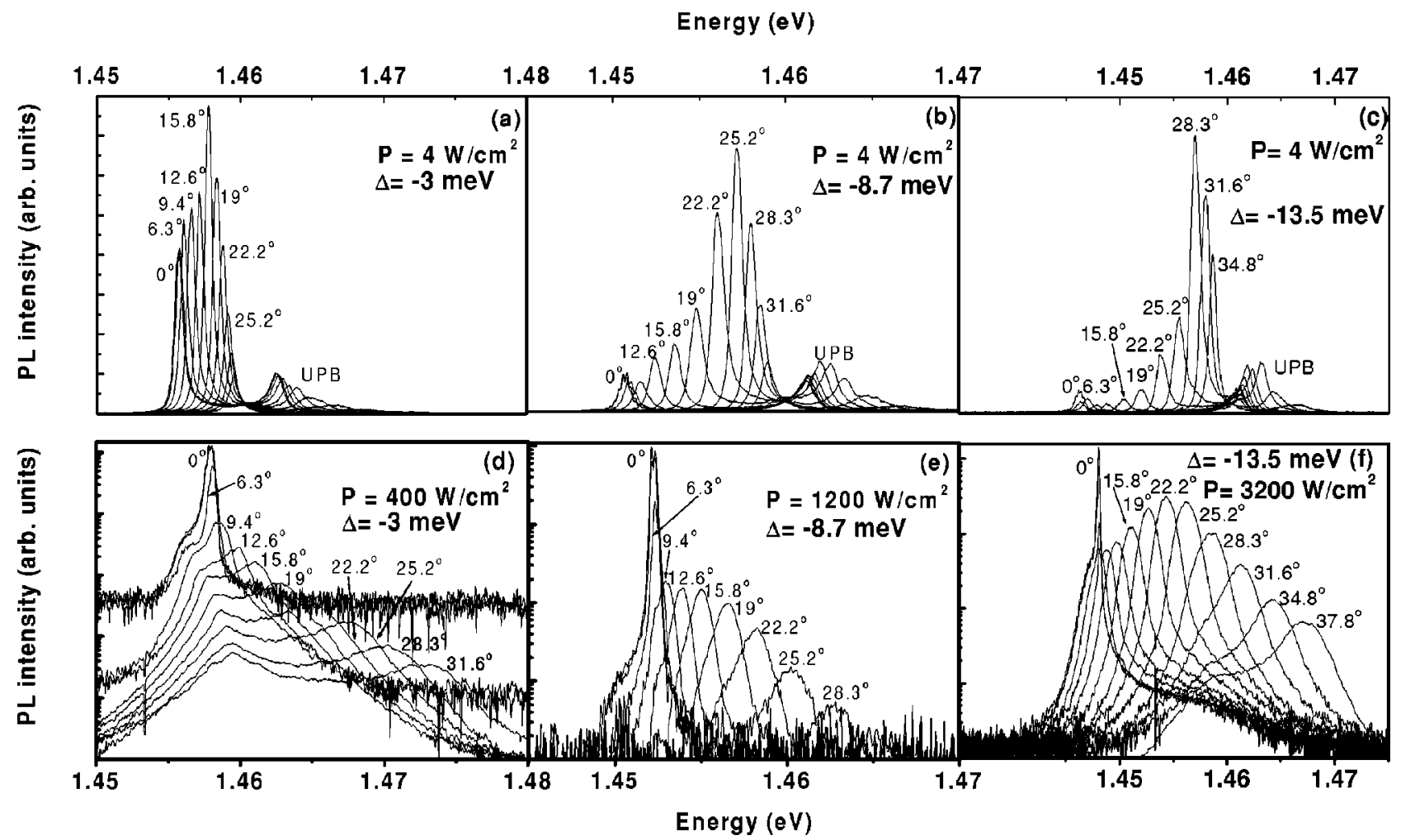

FIG. 4. Angular dependent emission spectra at low powers of $4 \mathrm{~W} / \mathrm{cm}^{2}$ for $\Delta=-3,-8.7$, and $-13.5 \mathrm{meV}$ in (a) -(c) and above threshold in (d)-(f). Ring emission arising from the relaxation bottleneck is observed at low power, with the maximum in intensity shifting to higher angle with increasing negative detuning. Above threshold the PL shows the sharp stimulated peak at $\theta=0$ in each case. At $\Delta=-3$ and $-8.7 \mathrm{meV}$, the PL decreases in intensity with angle, showing that the bottleneck is suppressed, whereas at $-13.5 \mathrm{meV}$, the PL maximum still occurs at finite angle even in the presence of the stimulation.

states relative to those at higher $k$ is a manifestation of the relaxation bottleneck for polaritons, ${ }^{6-9}$ discussed in the Introduction, and shown schematically in Fig. 1(a). We also note that since the microcavities have two-dimensional inplane symmetry, the peaking of the PL intensities at a particular nonzero value of $\theta$ corresponds to ring emission in the two-dimensional plane (such two-dimensional PL images under near resonant excitation are presented by Savvidis et $\left.a l .{ }^{19}\right)$. It is also notable that when corrected for the photon fraction of the states involved (Fig. 5), the depletion of the low $k$ states is seen to be very pronounced, with populations $\sim 5,10$, and 50 times smaller in Figs. 5(a)-5(c), respectively than those of the high $k$ states. It is also important to note that once the maximum populations as a function of $k$ are reached in Fig. 5, the relative populations remain approximately independent of $k$ to higher $k$, as the states of the exciton reservoir are approached.

At the powers above threshold, in Figs. 4(d)-4(f) the behavior is very different, with in each case the very sharp peak of Fig. 2 occurring at $k=0$. At $\Delta=-3$ and $-8.7 \mathrm{meV}$, the

\section{$\theta$ (Degrees)}
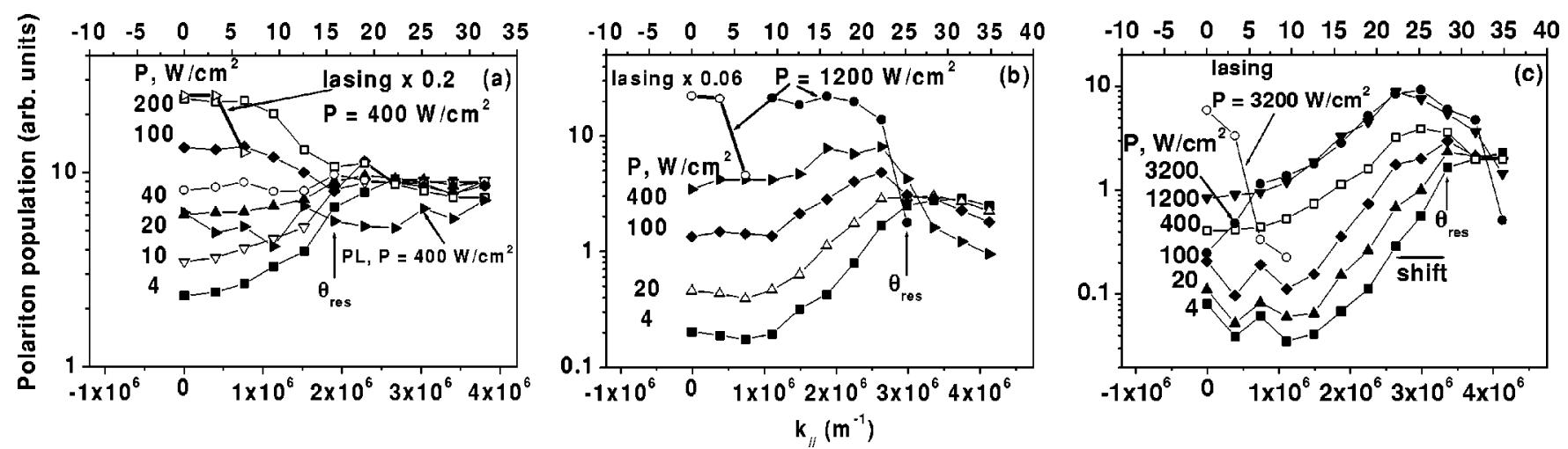

FIG. 5. (a)-(c) Polariton populations, obtained from results of Fig. 4 for $\Delta=-3,-8.7$, and $-13.5 \mathrm{meV}$ as a function of angle (and in-plane wave vector). The bottleneck is suppressed well below threshold in (a), only very close to threshold in (b), whereas in (c) at $\Delta$ $=-13.5 \mathrm{meV}$, the low $k$ states are still depleted relative to those at high $k$ even above the stimulation threshold. 
PL intensities now decrease with angle from low to high $k$, whereas at $-13.5 \mathrm{meV}$, following the sharp peak at $k=0$, the PL intensity exhibits a secondary maximum at $\sim 22^{\circ}$. The whole range of power dependent results, corrected for the photon fractions of the radiating states, are presented in Figs. 5(a)-5(c). We consider each of the detunings in turn, since the behavior has significant differences with increasing detuning

(a) $\Delta=-3 \mathrm{meV}$. The depletion of the low $k$ states is suppressed at incident powers of $\sim 40 \mathrm{~W} / \mathrm{cm}^{2}$, as we reported previously in Ref. 6 for a similar detuning. For higher powers the $k=0$ signal becomes stronger than that at high $k$, and then at $400 \mathrm{~W} / \mathrm{cm}^{2}$ the sharp stimulated emission is observed.

(b) $\Delta=-8.7 \mathrm{meV}$. Similar behavior to that at $-3 \mathrm{meV}$ is seen, but the low $k$ depletion is only fully suppressed at the onset of stimulated emission at $800 \mathrm{~W} / \mathrm{cm}^{2}$.

(c) $\Delta=-13.5 \mathrm{meV}$. With increasing power, the bottleneck shifts to lower $k$ by $\sim 6^{\circ},{ }^{20}$ but still with marked depletion of the low $k$ states even at the onset of stimulated emission. Accompanying the shift of the distribution towards lower $k$ there is a corresponding relative depletion of the high $k$ states (also visible at $-8.7 \mathrm{meV}$ detuning).

Thus to summarize the observations of Figs. 4 and 5, a marked relaxation bottleneck is observed for all the negative detunings at low power. At zero detuning, not presented here, there is no bottleneck, ${ }^{6,7}$ probably because of efficient population of the $k=0$ states by disorder scattering from the reservoir, or possibly also due to electron-exciton scattering. ${ }^{10}$ With increasing power the bottleneck is fully suppressed at $-3 \mathrm{meV}$ and very nearly at $-8.7 \mathrm{meV}$, before the onset of stimulated emission, by polariton-polariton and polaritonexciton and exciton-exciton scattering from states at higher $k$ to those at lower $k$ (see next section). ${ }^{21}$ At large negative detuning, the bottleneck is never suppressed before the onset of stimulated emission, due to the low exciton fraction of the low $k$ states (at $\Delta=-13.5 \mathrm{meV}$, the exciton fraction of the $k=0$ states is only 0.04 ), and probably also because of the larger energy loss required to populate the low $k$ states.

\section{Numerical simulation of the polariton relaxation in the strong coupling regime}

In order to place the interpretation of the above results on a firm basis, the polariton relaxation dynamics were modelled by solution of the Boltzmann equations for the polariton population $n_{k}$ in two-dimensional reciprocal space

$$
\begin{aligned}
\frac{d n_{k}}{d t}= & P_{k}-\Gamma_{k} n_{k}-n_{k} \sum_{k^{\prime}} W_{k \rightarrow k^{\prime}}\left(n_{k^{\prime}}+1\right) \\
& +\left(n_{k}+1\right) \sum_{k^{\prime}} W_{k^{\prime} \rightarrow k} n_{k},
\end{aligned}
$$

where $P_{k}$ is the generation term describing optical pumping, $\Gamma_{k}$ is the recombination rate composed of both radiative and nonradiative components, $W_{k \rightarrow k^{\prime}}$ is the total scattering rate between the states $k$ and $k^{\prime}$. This scattering rate is composed of two terms in our model, namely, polariton-acoustic phonon scattering and polariton-polariton scattering. An impor- tant assumption is that the generation term and, consequently, the polariton distribution in reciprocal space have cylindrical symmetry at all times considered. This is consistent with the experimental configuration of nonresonant excitation far from the anticrossing region. Also, direct generation of hot excitons by the incident laser pulse has been assumed, thus neglecting the kinetics of exciton formation from electron-hole pairs. Elastic scattering due to structural disorder is also neglected since it does not break the cylindrical symmetry of the system.

The recombination rate $\Gamma_{k}$ is defined as a function of $k$ in the following way. ${ }^{22}$

(a) For polariton wave-vectors within the stop band ( $k$ $<k_{s}$ ), we assume $\Gamma_{k}=c_{k} / \tau_{c}$, where $c_{k}$ is the photon fraction of the polariton and $\tau_{c}$ is the cavity photon lifetime (1.3 ps). $k_{s}=8 \times 10^{6} \mathrm{~m}^{-1}$ for our structure. In this region, excitons are strongly coupled with cavity-confined photons.

(b) For wave vectors outside the strong coupling regime but within the "light cone" (i.e., wave vectors less than that of the incident light in the medium, $k_{s}<k<k_{\text {light }}$ ) we assume $\Gamma_{k}=\Gamma_{0}$, with $\Gamma_{0}$ the radiative decay rate of free QW excitons taken as $\Gamma_{0}^{-1}=16 \mathrm{ps}^{23}$ In this region of reciprocal space, the QW excitons are weakly coupled with the continuum of free photon modes. The lifetime taken for excitons in this region has very little effect on the overall polariton dynamics.

(c) Outside the "light cone," i.e., for $k_{\text {light }}<k, \Gamma_{k}=1 / \tau_{e}$ is assumed, where $\tau_{e}$ is the exciton (nonradiative) lifetime taken to be $\sim 0.5 \mathrm{~ns}^{23}$ In this region, the exciton reservoir, scattering processes to lower $k$ are needed in order for radiative recombination to occur. We calculate the polaritonacoustic phonon and polariton-polariton scattering rates following Tassone, ${ }^{8,9}$ including scattering only within the lower-polariton branch. We neglect in this model all spinrelated effects, with the matrix element for exciton-exciton scattering calculated assuming parallel exciton spins.

Figure 6 shows the polariton population calculated as a function of emission angle from solution of Eq. (1) for $\Delta=-3 \mathrm{meV}$ (a), $-8.7 \mathrm{meV}$ (b), and $-13.5 \mathrm{meV}$ (c), for different values of the excitation power (to be compared with the experimental data in Fig. 5). Comparison of Figs. 6 and 5 shows that the main features of the experimental spectra up to $\sim 100 \mathrm{~W} / \mathrm{cm}^{2}$ are reproduced very well by the theory. ${ }^{24}$ The suppression of the bottleneck with increasing excitation intensity at small negative detuning in Fig. 5(a) is seen clearly in Fig. 6(a), with good quantitative agreement between theory and experiment for the power $\left(30-40 \mathrm{~W} / \mathrm{cm}^{2}\right)$ at which the bottleneck is suppressed being found. ${ }^{25}$ The trend of weaker suppression of the bottleneck with increasing detuning in Figs. 5(b), 5(c) is also found in Figs. 6(b), $6(c)$ as the exciton fraction of the low $k$ states decreases. Furthermore, at large negative detuning, the shift of the maximum of the distribution in Fig. 5(c) to lower $k$ with power is also reproduced well in the theory of Fig. 6(c). The good overall agreement between theory and experiment thus confirms the dominant role of exciton-exciton scattering processes in controlling the dynamics of the cavity polaritons 

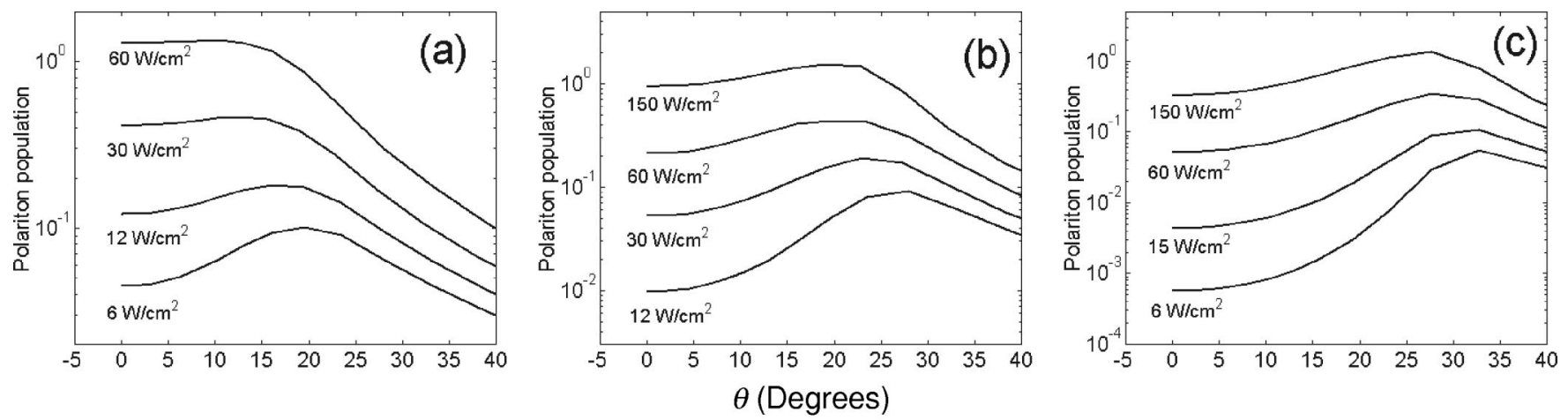

FIG. 6. (a)-(c) Polariton populations obtained from numerical solution of Eq. (1) for detunings of (a) -3 , (b) $=-8.7$, and (c) $-13.5 \mathrm{meV}$ as a function of angle for a range of excitation intensities.

under conditions of nonresonant excitation, for intensities greater than $\sim 5 \mathrm{~W} / \mathrm{cm}^{2}$ (phonon scattering is dominant at lower powers).

The maximum excitation intensity shown for each detuning in the calculations is at least a factor of 2 less than the powers at which the transition from the strong to the weak coupling regime is observed experimentally (see Sec. III D for details on the loss of strong coupling). We can thus be confident that the comparison between theory and experiment is being made in the strong coupling regime where the theoretical treatment is applicable. It is notable that occupancies of order unity are predicted at the highest powers in Figs. 6(a)-6(c), the condition required for the onset of stimulated scattering. However, the simulations also show that the populations only begin to increase exponentially at powers 2-3 higher than those shown in Fig. 6, in regimes where strong coupling is found to be lost experimentally (see Sec. III D). Thus the simulations, as well as the experiments (see Fig. 3) correspond to the regime of exciton-exciton pair scattering with at most a quadratic dependence of population on power.

Although the theory accounts well for the polariton distribution and its dependence on power in the strongly coupled regime, there are qualitative differences in the regime of large wave vectors, beyond the resonance $k$ values in Figs. 5, 6 . The theory predicts decreasing occupancies beyond resonance, whereas in experiment occupancies nearly independent of $k$ for $k>k_{\text {res }}$ are found. The higher occupation in experiment probably arises from additional quasielastic disorder scattering into states close in energy to the exciton reservoir, the same mechanism very likely giving rise to the absence of the bottleneck at zero detuning, as discussed in the Introduction.

\section{Dispersion curves at low power and above threshold, the transition from weak to strong coupling}

It is seen from the above that at low power a relaxation bottleneck is observed for all the negative detunings, which is either fully or partially suppressed before the onset of stimulated emission from the cavity. We now present the measured dispersions as a function of angle to answer the question posed in the introduction as to whether the stimulated emission occurs in the strong or weak coupling regimes for all the detunings. ${ }^{26}$ The peak energies as a function of angle are presented in Fig. 7 for the three detunings, at low power $\left(4 \mathrm{~W} / \mathrm{cm}^{2}\right)$ and at powers just above the stimulation threshold. At low power, the characteristic anticrossing of the strong coupling regime is seen with the resonance angle $\theta_{\text {res }}$ shifting from $13^{\circ}$ to $25^{\circ}$ to $29^{\circ}$ for $-3,-8.7$, and -13.5 $\mathrm{meV}$ detunings. It is notable that in each case, the peak in the PL intensity distribution with $\theta$ in Figs. 4(a)-4(c) occurs to within $2^{\circ}$ at $\theta_{\text {res }}$. This arises since the density of polariton states decreases strongly below $\theta_{\text {res }}$, as the states become increasingly photonlike with effective mass tending towards
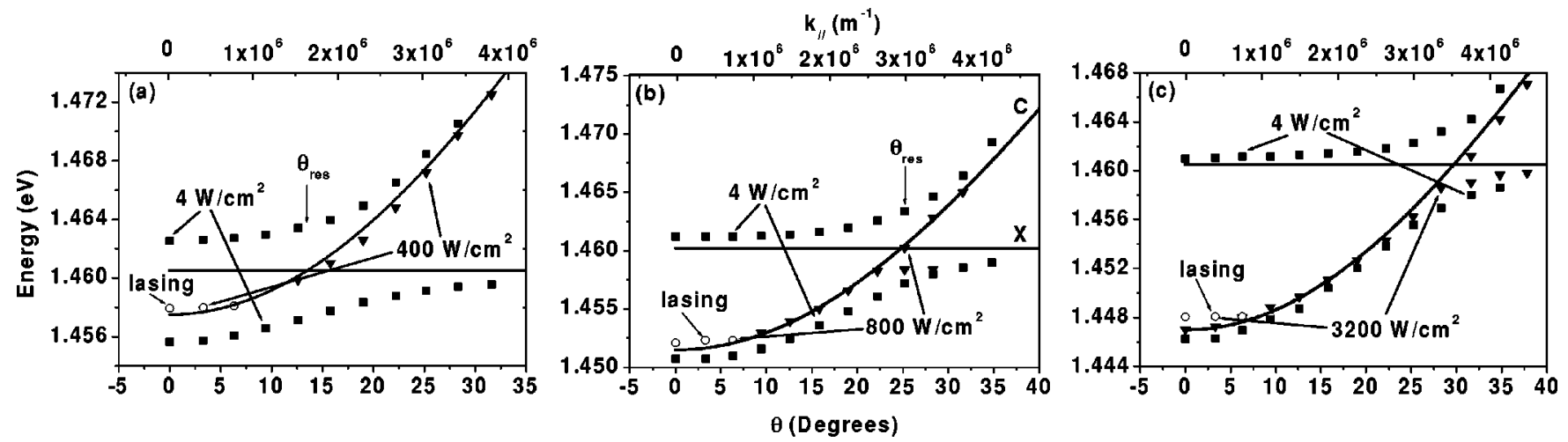

FIG. 7. (a)-(c) Measured dispersions well below (squares) and above threshold (triangles) for $\Delta=-3,-8.7$, and -13.5 meV. In each case above the stimulation threshold, the emission peaks occur at the energy of the uncoupled cavity mode in the weak coupling regime. The full curves are the calculated exciton $(X)$ and cavity $(C)$ dispersions. 


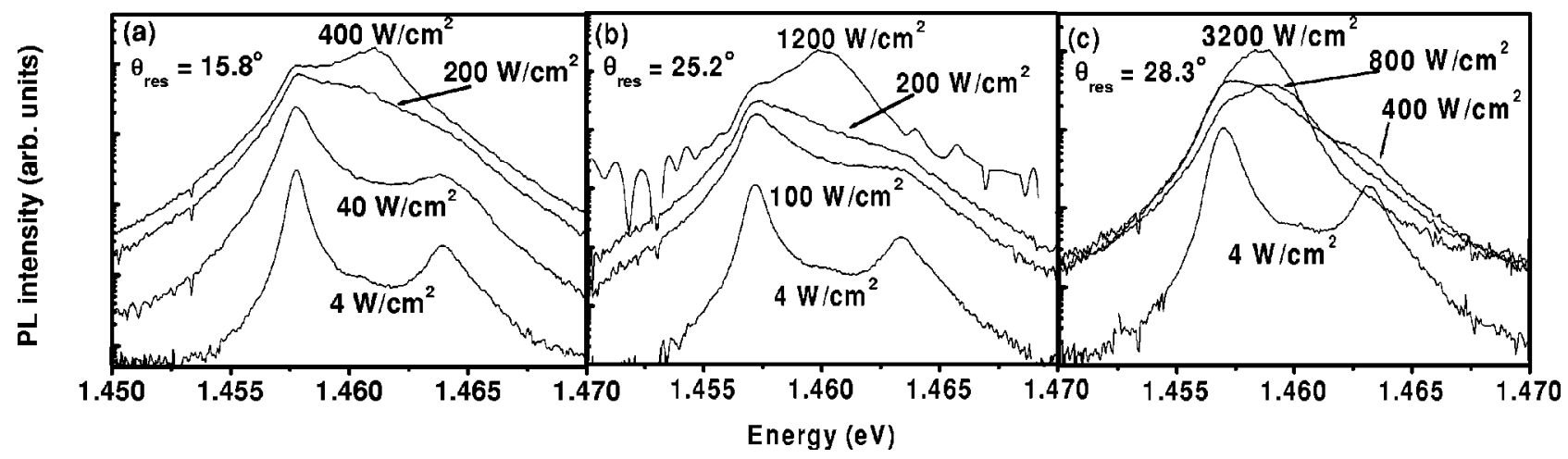

FIG. 8. (a)-(c) Emission spectra as a function of power for $\Delta=-3,-8.7$, and $-13.5 \mathrm{meV}$. In each case the two peaked polariton spectrum in the strong coupling regime collapses into the single emission line at the cavity mode energy below the thresholds for stimulation ( $P_{\text {stim }}=400,800$, and $1600 \mathrm{~W} / \mathrm{cm}^{2}$ in the three cases, respectively).

the photon mass of $3 \times 10^{-5} m_{e}$, thus leading to the occurrence of the bottleneck for states at $\theta<\theta_{\text {res }}{ }^{7}$

Furthermore it is seen that above threshold, in each case the measured dispersion follows closely that of the uncoupled cavity mode, and thus that stimulation is only reached when the structures are in the weak coupling regime. At $\Delta=-3 \mathrm{meV}$, it is already clear from the $k=0$ peak position that weak coupling has been reached, with the stimulated peak occurring $\sim 2 \mathrm{meV}$ above the LPB energy, close to the energy of the uncoupled cavity mode. For the larger negative detunings the $k=0$ situation is less clear with peak shifts of $<1 \mathrm{meV}$ found from below to above threshold (as expected since at larger negative detuning the LPB energy lies close to that of the uncoupled cavity mode). The occurrence of weak coupling above threshold is made fully clear, however, from the overall variation with $k$ which follows closely that of the uncoupled cavity mode, particularly in the resonance regime where the two peaked structure characteristic of strong coupling at low power is replaced by one peak above threshold at the cavity mode energy.

The transition from weak to strong coupling is most clearly examined from the spectra at the resonance angles for the three detunings as a function of power shown in Fig. 8. The transition from the strong-coupling two polariton peaks at low power, to the single peak at high power is again seen. The topmost spectrum in each case is taken just above the stimulation threshold. It is notable, however, that for each detuning the two peaked structure disappears at factors of 2 $(\Delta=-3 \mathrm{meV})$ to $8(\Delta=-13.5 \mathrm{meV})$ lower power than the stimulation thresholds, at power densities in the range from 200 to $400 \mathrm{~W} / \mathrm{cm}^{2}$. Thus it is fully clear from the results of Figs. 7, 8 that stimulation is only reached at powers beyond which the strong coupling has already been lost.

Further information on why the strong coupling is lost at the observed powers is given from the variation of the lower polariton branch linewidths as a function of angle in Fig. 9. At low power nearly angle independent linewidths of $\sim 0.5$ at $-3 \mathrm{meV}, 0.7$ at $-8.7 \mathrm{meV}$, and 0.9 at $-13.5 \mathrm{meV}$ are observed (the increasing width with detuning is due to the exciton inhomogeneous broadening, as discussed in Ref. 27). At high power the variation is very different, with a strong increase of linewidth with angle being found. ${ }^{28}$ The increase of linewidth as the high $k$ exciton states are approached is a clear signature of the onset of exciton-exciton scattering and exciton screening at high density. Indeed it is notable that at the high powers shown the measured linewidths at high $k$ are $\geqslant 4 \mathrm{meV}$, of the order of the normal mode splitting of 6 $\mathrm{meV}$, consistent with the loss of strong coupling.

Precise conversion of the power density at which the strong coupling is lost to photocreated exciton density is made difficult by a number of experimental uncertainties. As noted at the start of Sec. III A, the power densities quoted are estimated powers absorbed in the cavity. Assuming a typical exciton lifetime of $0.5 \mathrm{nsec},{ }^{23}$ the power density at which
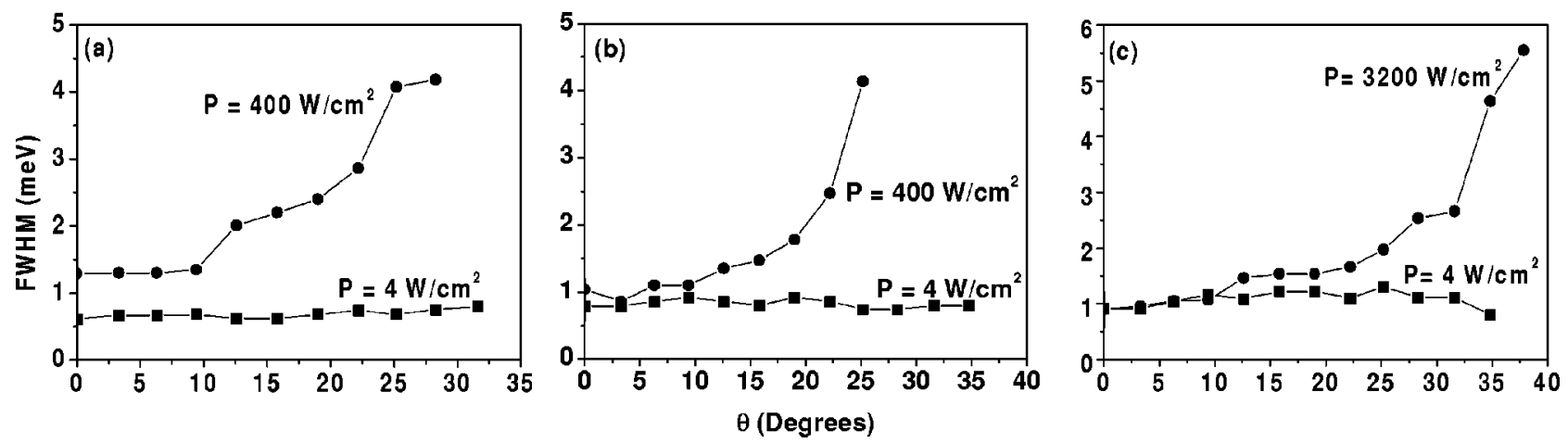

FIG. 9. (a)-(c) Lower polariton branch linewidths (full width half maximum) for $\Delta=-3,-8.7$, and -13.5 meV at low power $\left(4 \mathrm{~W} / \mathrm{cm}^{2}\right)$ and at high power close to or above threshold. 


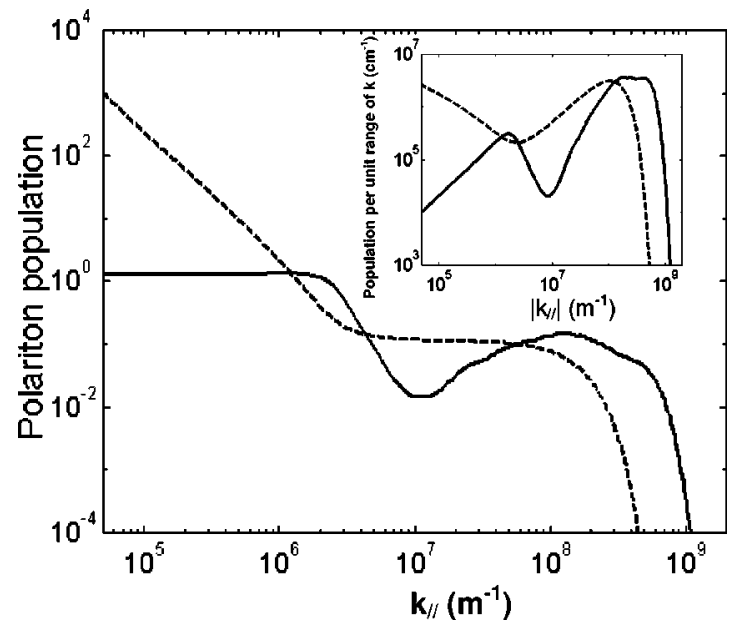

FIG. 10. Calculated exciton-polariton population as a function of wave vector, obtained from numerical solution of Eq. (1) for $\Delta$ $=-3 \mathrm{meV}$ and excitation intensity $60 \mathrm{~W} / \mathrm{cm}^{2}$ (solid line). Equilibrium polariton population given by Bose statistics with zero chemical potential (dashes). The inset shows the population per unit range of $k$ around a ring of wavevector $k N_{\text {ring }}(k) / d k$ from the kinetic equation (solid line) and for equilibrium Bose statistics (dashes) for the same set of parameters as in the main part of the figure.

strong coupling is lost of $200 \mathrm{~W} / \mathrm{cm}^{2}$ in Figs. 7(a) and 7(b) [400 W/ $\mathrm{cm}^{2}$ in Fig. 7(c)] corresponds to an exciton sheet density of $4 \times 10^{11} \mathrm{~cm}^{-2}$. Since there are six quantum wells in the sample, strong coupling is thus lost at a density of 7 $\times 10^{10} \mathrm{~cm}^{-2}$ per well, close to the Mott limit for excitons in GaAs QWs, and consistent with early work of Houdré et al. ${ }^{26}$ and experiment and calculations of Kira et al. ${ }^{15}$ Lasing occurs at factors of 2, 4, and 8 higher densities at the three detunings studied, clearly in the weak coupling regime, the increase of threshold power with detuning arising from the decreasing overlap of the cavity mode with the gain curve of the light emitting quantum well excitons. ${ }^{30}$

It is thus clear in both experiment and theory that sufficiently large low $k$ populations are not achieved for stimulation to occur before strong coupling is lost. We have also shown from the experiment-theory comparisons that the relatively small polariton populations arise from the inefficient relaxation from the reservoir into the polariton region. Further insight into the mechanisms underlying the loss of strong coupling is given by the calculated polariton population over a large range of $k$ shown in Fig. 10, obtained by solution of Eq. (1) for $\Delta=-3 \mathrm{meV}$ and a pumping intensity of $60 \mathrm{~W} / \mathrm{cm}^{2}$. In the main part of the figure the population per state along a line in $k$-space is shown (the full line), as in Figs. 5 and 6 , and in the inset the population per unit range of $k$ around a ring of wave vector $k\left[N_{\text {ring }}(k) / d k\right]$ is presented. ${ }^{29}$ The total polariton density is then given by

$$
N_{\text {tot }}=\int_{0}^{+\infty} \frac{1}{(2 \pi)^{2}} n_{k} 2 \pi k d k .
$$

Since the system has cylindrical symmetry, this favours large $k$ relative to low $k$ states, as compared to the depen- dence along a line, as a result of the strongly increasing density of states with $k$. The distributions from Eq. (1) are compared with the equilibrium distribution function (the dashes) expected for a Bose gas of polaritons with zero chemical potential $(\mu)$. The critical density for condensation (and hence $\mu=0$ ) is calculated to be $2.4 \times 10^{10} \mathrm{~cm}^{-2}$ at 25 $\mathrm{K}$, much smaller than the density employed for the full curve of Fig. 10 calculated to be $1.5 \times 10^{11} \mathrm{~cm}^{-2}$ [the small density for $\mu=0$ is due to the small polariton mass within the trap and also to the small lateral size of the optically excited area $(25 \mu \mathrm{m})]$.

There are several major differences between the calculated "kinetic" distribution and the thermal equilibrium one. First, there is strong deviation at small $k$, a direct consequence of the bottleneck effect, as discussed earlier. Moreover, at high $k$ greater than $\sim 10^{8} \mathrm{~m}^{-1}$, the kinetic distribution lies substantially higher than the equilibrium distribution, demonstrating the extra population of the reservoir, due to the inefficient relaxation, the large reservoir population being a key factor in the loss of strong coupling before stimulation is achieved. Finally the deep minimum in the solid curve in Fig. 10 at $k \sim 10^{7} \mathrm{~m}^{-1}$ arises from the efficient exciton-exciton scattering in this region: one exciton of the pair loses energy to provide a nonzero population below the bottleneck, with the other member scattering to high $k$ to populate the reservoir, eventually giving rise to exciton screening at high density. ${ }^{31}$

\section{CONCLUSION}

We have shown both experimentally and theoretically that the occurrence of the polariton relaxation bottleneck plays a major role in inhibiting the efficient population of low wave vector polariton states, under conditions of nonresonant excitation. Even though the density of states in this region is low as a result of the very low polariton mass, the relatively slow relaxation rates relative to the polariton lifetimes prevent polariton occupancies greater than unity from being achieved in the GaAs-based microcavities under study, before the onset of exciton screening. The stimulation phenomena under conditions of nonresonant excitation occur instead in the weak coupling regime with uncoupled exciton and photon modes. This contrasts strongly with the situation of resonant excitation where the polariton region is populated directly by the pump laser, and the relaxation bottleneck is bypassed and high population of the exciton reservoir is avoided.

The prospects for the observation of polariton lasing, under conditions of nonresonant excitation nevertheless remain good in systems of higher exciton binding energy such as II-VI materials, ${ }^{32} \mathrm{GaN}$, or organics. ${ }^{33}$ In this case sufficiently strong population of the low $k$ states by exciton-exciton scattering before the onset of exciton screening may be expected due to the greater resistance of the exciton states to screening, although the greater energy loss required from the exciton reservoir to populate $k=0$ states will be an inhibitory factor to the achievement of high populations. Indeed the early report ${ }^{32}$ of a stimulated peak close to the energy of the lower polariton branch in CdTe microcavities under nonreso- 
nant excitation conditions provides optimism that such polariton lasing may indeed be achievable. A further mechanism of high potential ${ }^{10}$ to achieve polariton lasing in systems of lower exciton binding energy is to employ microcavities with excess electron doping in the quantum wells, but doped at densities below the screening limit. Under such conditions the additionnal exciton-electron scattering channel is expected to lead to very effective population of the low $k$ states at relatively low exciton densities in the region of $\sim 10^{10} \mathrm{~cm}^{-2}$.

\section{ACKNOWLEDGMENTS}

We acknowledge the support of EPSRC and the European Community Human Potential Program, Contract No. HPRNCT-1999-00132, CLERMONT, and valuable interactions with D. M. Whittaker, M. Emam-Ismail, and A. Lemaitre.
${ }^{1}$ For reviews see M.S. Skolnick, T.A. Fisher, and D.M. Whittaker, Semicond. Sci. Technol. 13, 645 (1998); G. Khitrova, H.M. Gibbs, F. Jahnke, M. Kira, and S.W. Koch, Rev. Mod. Phys. 71, 1591 (1999).

${ }^{2}$ R.M. Stevenson, V.N. Astratov, M.S. Skolnick, D.M. Whittaker, M. Emam-Ismail, A.I. Tartakovskii, P.G. Savvidis, J.J. Baumberg, and J.S. Roberts, Phys. Rev. Lett. 85, 3680 (2000).

${ }^{3}$ P.G. Savvidis, J.J. Baumberg, R.M. Stevenson, M.S. Skolnick, D.M. Whittaker, and J.S. Roberts, Phys. Rev. Lett. 84, 1547 (2000)

${ }^{4}$ J.J. Baumberg, P.G. Savvidis, R.M. Stevenson, A.I. Tartakovskii, M.S. Skolnick, D.M. Whittaker, and J.S. Roberts, Phys. Rev. B 62, R16 247 (2000).

${ }^{5}$ Possible evidence for stimulated polariton scattering under nonresonant excitation conditions has been reported in II-VI microcavities by Le. Si Dang, D. Heger, R. André, F. Boeuf, and R. Romestain, Phys. Rev. Lett. 81, 3920 (1998).

${ }^{6}$ A.I. Tartakovskii, M. Emam-Ismail, R.M. Stevenson, M.S. Skolnick, V.N. Astratov, D.M. Whittaker, J.J. Baumberg, and J.S. Roberts, Phys. Rev. B 62, R2283 (2000).

${ }^{7}$ M. Müller, J. Bleuse, and R. Andr, Phys. Rev. B 62, 16886 (2000).

${ }^{8}$ F. Tassone, C. Piermarocchi, V. Savona, A. Quattropani, and P. Schwendimann, Phys. Rev. B 56, 7554 (1997).

${ }^{9}$ F. Tassone and Y. Yamamoto, Phys. Rev. B 59, 10830 (1999).

${ }^{10}$ A.V. Kavokin, G. Malpuech, A. Di Carlo, and J.J. Baumberg, Phys. Stat. Solidi A (to be published).

${ }^{11}$ P. Senellart and J. Bloch, Phys. Rev. Lett. 82, 1233 (1999).

${ }^{12}$ A. Imamoğlu and R.J. Ram, Phys. Lett. A 214, 193 (1996).

${ }^{13}$ S. Pau, H. Cao, J. Jacobson, G. Björk, and Y. Yamamoto, Phys. Rev. A 54, R1789 (1996).

${ }^{14}$ H. Cao, S. Pau, J.M. Jacobson, G. Björk, Y. Yamomoto, and A. Imamoğlu, Phys. Rev. A 55, 4632 (1997).

${ }^{15}$ M. Kira, F. Jahnke, S.W. Koch, J.D. Berger, D.V. Wick, T.R. Nelson, G. Khitrova, and H.M. Gibbs, Phys. Rev. Lett. 79, 5170 (1997).

${ }^{16}$ The width of the lasing mode at $-3 \mathrm{meV}$ detuning is greater than at -8.7 and $-13.5 \mathrm{meV}$. Although we have no definitive explanation for this behavior, it may arise from a small instability in the lasing energy at $-3 \mathrm{meV}$ just above threshold.

${ }^{17}$ Although our measurements were performed without strong spatial selection we can see from the data of Fig. 2 (presented on a logarithmic scale) that the shoulders visible at high power densities correspond to PL emission much weaker than the main emission line ( 5 to $10 \%$ maximum), showing that the PL detected comes predominantly from a region of uniform excitation.
${ }^{18}$ P. Senellart, J. Bloch, B. Sermage, and J.Y. Marzin, Phys. Rev. B 62, R16 263 (2000).

${ }^{19}$ P.G. Savvidis, J.J. Baumberg, D. Porras, D.M. Whittaker, M.S. Skolnick, and J.S. Roberts, Phys. Rev. B 65, 073309 (2002).

${ }^{20}$ This behavior corresponds to the shifting of the PL maximum at $28^{\circ}$ in Fig. 3(c) to $22^{\circ}$ in Fig. 3(f).

${ }^{21}$ In Ref. 6 we presented evidence that an important component of such scattering arose from a pair process, in which one polariton scatters down in energy with a second one scattering up in energy to conserve energy and momentum.

${ }^{22}$ For a fuller discussion of the three regions see, e.g., J. Bloch and J.Y. Marzin, Phys. Rev. B 56, 2103 (1997).

${ }^{23}$ B. Sermage, S. Long, I. Abram, J.Y. Marzin, J. Bloch, R. Planel, and V. Thierry-Mieg, Phys. Rev. B 53, 16516 (1996).

${ }^{24}$ The theoretical spectra shown are restricted to powers of $\sim 100 \mathrm{~W} / \mathrm{cm}^{2}$, since as shown in Sec. III D strong coupling is lost at higher powers, and the theory which assumes strongly coupled exciton-photon states is no longer applicable.

${ }^{25}$ Given the many uncertainties in the experimental determination of the power absorbed in the cavity discussed in Sec. III A, and, for example, in the value of the exciton lifetime, the main adjustable parameter employed in the theory, the quantitative agreement is probably in part fortuitous.

${ }^{26}$ The transition from strong to weak coupling was studied by angular dependent spectroscopy by R. Houdré, J.L. Gibernon, P. Pellandini, R.P. Stanley, U. Oesterle, C. Weisbuch, J. O'Gorman, B. Roycroft, and M. Ilegems, Phys. Rev. B 52, 7810 (1995).

${ }^{27}$ A.V. Kavokin, Phys. Rev. B 57, 3757 (1998).

${ }^{28}$ In Ref. 6 a similar increase of linewidth with angle at an intermediate power corresponding to the collapse of the bottleneck for $-4 \mathrm{meV}$ detuning was found.

${ }^{29} N_{\text {ring }}(k) / d k=\left[1 /(2 \pi)^{2}\right] n_{k} 2 \pi k$ where $1 /(2 \pi)^{2}$ is a normalization constant.

${ }^{30}$ X. Fan, H. Wang, H.Q. Hou, and B.E. Hammons, Phys. Rev. B 56, 15256 (1997) claim the observation of lasing below the bleaching threshold for negative detunings where the cavity mode is resonant with localised excitons. However, the angular dependent studies we report show that strong coupling is lost before lasing under such conditions.

${ }^{31}$ As discussed at the end of Sec. III C, this minimum is suppressed in experiment probably due to disorder scattering to states at lower $k$.

${ }^{32}$ See Ref. 5.

${ }^{33}$ D.G. Lidzey, D.D.C. Bradley, M.S. Skolnick, T. Virgili, S. Walker, and D.M. Whittaker, Nature (London) 395, 53 (1998); D.G. Lidzey, D.D.C. Bradley, T. Virgili, A. Armitage, M.S. Skolnick, and S. Walker, Phys. Rev. Lett. 82, 3316 (1999). 